\title{
Perspectives on the mechanism of action and clinical application of eribulin for metastatic breast cancer
}

\author{
Joyce O'Shaughnessy*,1, Virginia Kaklamani² \& Kevin Kalinsky ${ }^{3}$ \\ ${ }^{1}$ Baylor University Medical Center, Texas Oncology, US Oncology, Dallas, TX, USA \\ ${ }^{2}$ Department of Medicine, Division of Hematology/Oncology, University of Texas Health Science Center San Antonio, San Antonio, \\ TX, USA \\ ${ }^{3}$ Columbia University Irving Medical Center, NY, USA \\ *Author for correspondence: Tel.: +1 214370 1000; Fax: +1 214370 1850; joyce.oshaughnessy@usoncology.com
}

Eribulin is a novel microtubule inhibitor with mitotic and nonmitotic mechanisms of action. Both pooled and subgroup analyses from large-scale Phase III clinical trials demonstrated that eribulin has substantial activity in patients with pretreated (anthracycline and a taxane) advanced or metastatic breast cancer. We review recent pharmacological and clinical findings pertaining to eribulin use in metastatic breast cancer - particularly highlighting eribulin in difficult-to-treat and aggressive disease, and safety data in specific patient populations. Additionally, recent advancements in our understanding of the mechanism of action of eribulin and potential future directions for its clinical development are discussed. Ongoing studies of eribulin in combination with immunotherapies and established cytotoxic agents may help shape the future landscape of breast cancer treatment.

Lay abstract: Eribulin, a nontaxane microtubule inhibitor, is approved for the treatment of metastatic breast cancer. In this review, we present the clinical activity and safety of eribulin in metastatic breast cancer - highlighting studies investigating efficacy and tolerability in some aggressive, difficult-to-treat breast cancer populations.

First draft submitted: 17 December 2018; Accepted for publication: 19 February 2019; Published online: 20 March 2019

Keywords: eribulin $\bullet$ mechanism of action $\bullet$ metastatic breast cancer $\bullet$ microtubule inhibitor $\bullet$ triple-negative breast cancer

Breast cancer is the most common cancer in women, with more than 260,000 new cases and over 40,000 deaths expected in the USA in 2018 [1]. In the USA, about 6\% of patients are expected to present with metastatic disease [1], and a number of patients with early-stage disease develop metastases after primary breast cancer treatment. Metastatic breast cancer $(\mathrm{MBC})$ remains difficult to treat, with a 5-year survival rate of about $27 \%$ [1].

Eribulin, a novel inhibitor of microtubule dynamics, is used in the USA, Europe and other countries worldwide for the treatment of certain patients with advanced or MBC who are refractory to other treatments [2-4]. In the USA, eribulin is approved for patients with $\mathrm{MBC}$ who have previously received $\geq 2$ chemotherapy regimens, including an anthracycline and a taxane in either the adjuvant or metastatic setting [4]. In the European Union, eribulin is currently indicated for the treatment of patients with locally advanced or MBC who have received $\geq 1$ chemotherapy regimen(s), including an anthracycline and a taxane in either the adjuvant or metastatic setting [5].

Eribulin mesylate is a structurally simplified synthetic analog of halichondrin B, a natural product that is isolated from the marine sponge Halichondria okadai [2]. As a member of the halichondrin class of antineoplastic drugs, eribulin is an inhibitor of microtubule dynamics with a unique tubulin-based mechanism of action (MOA) that results in inhibition of the growth phase of the microtubule, without any corresponding inhibition of the shortening phase [6-8].

Approval for eribulin was based on the efficacy and safety findings from the pivotal Phase III EMBRACE (Eisai Metastatic Breast Cancer Study Assessing Physician's Choice vs E7389; Study 305; NCT00388726) [9]. Results 
from this study showed a statistically significant overall survival (OS) advantage for eribulin versus treatment of physician's choice (TPC) in heavily pretreated patients with locally advanced or MBC (13.1 vs 10.6 months; hazard ratio [HR]: 0.81; 95\% CI: 0.66-0.99; $\mathrm{p}=0.041)$ [9].

A second, randomized, Phase III study (Study 301; NCT00337103) evaluated eribulin compared with capecitabine in patients receiving first-, second- or third-line chemotherapy for advanced or metastatic disease [10]. Although no significant OS benefit was demonstrated for eribulin in the overall study population when compared with capecitabine, a prespecified subgroup analysis suggested that eribulin provided an OS benefit in patients with HER2-negative disease [10]. Furthermore, a pooled analysis of data from EMBRACE and Study 301 showed a survival benefit for patients receiving eribulin versus active control (median OS: 15.2 vs 12.8 months; HR: 0.85; 95\% CI: $0.77-0.95 ; \mathrm{p}=0.003$ ) [11]. In this pooled analysis, there were OS improvements in particular patient groups for eribulin versus other treatments, including patients with HER2-negative disease (HR: 0.82; $\mathrm{p}=0.002$ ) and patients with triple-negative breast cancer (TNBC; HR: 0.74; $\mathrm{p}=0.006$ ) [11].

Since the initial approval of eribulin in the USA in 2010, numerous clinical investigations of eribulin, as either monotherapy or in combination with other antineoplastic agents, have been conducted in patients with MBC and other breast cancer types. Eribulin is the only monotherapy that has shown a significant OS benefit in patients who have received at least two prior lines of therapy $[10,12]$. This review will highlight recent pharmacological and clinical findings pertaining to eribulin use in MBC including: newer aspects of our understanding of eribulin's MOA; clinical data related to the use of eribulin in breast cancer (summarized in Table 1), with a particular focus on difficult-to-treat and aggressive disease; safety data highlighting eribulin as a unique cytotoxic agent; and potential future directions for the clinical development of eribulin.

\section{Advances in understanding eribulin's MOA}

The antimitotic MOA of eribulin occurs via inhibition of microtubule polymerization (or growth) by its binding to high-affinity sites on $\beta$-tubulin at the exposed (plus) ends of growing microtubules $[6,8,13]$. Unlike other antitubulin agents (e.g., taxanes, epothilones and vinca alkaloids), eribulin does not affect the shortening phase of microtubule dynamics $[6-8,14,15]$. In addition, eribulin triggers formation of nonproductive tubulin aggregates that potentially further inhibit mitotic spindle formation by lowering intracellular concentrations of free tubulin [6]. The end results of both processes are blockage of mitotic spindle formation, mitotic arrest and cell death by apoptosis [16]. A recent preclinical study demonstrated that eribulin causes loss of the tubulin polymerase ch-TOG from the growing (plus) ends of microtubules, contributing to alterations in microtubule dynamics and cancer cell migration [17].

In addition to its antimitotic mechanism, in preclinical cancer models, eribulin has shown nonmitotic effects including vascular remodeling, reversal of the epithelial-to-mesenchymal (EMT) transition and suppression of cancer cell migration, invasion and experimental metastasis [18-20]. In preclinical xenograft models of human breast cancer, eribulin caused tumor vascular remodeling leading to increased perfusion of tumor cores [19]. This was demonstrated by an increase in the tumor perfusion transfer coefficient, visually using dynamic contrast enhanced MRI, increased Hoechst dye perfusion and decreases in staining for carbonic anhydrase 9, an established marker of hypoxia. These effects were associated with increased numbers of microvessels in tumors that had been exposed to a single dose of eribulin approximately 1 week earlier. Furthermore, prior treatment with eribulin enhanced the antitumor activity of capecitabine in the MDA-MB-231 xenograft model. These findings suggest that eribulin-induced vascular remodeling leads to changes in the tumor microenvironment that reduce or eliminate hypoxia-driven growth aggressiveness and increase exposure of subsequent treatments.

Additional preclinical evidence further illustrates differences in eribulin's MOA compared with other microtubule inhibitors. For example, in a functional endothelial-pericyte coculture assay, eribulin demonstrated efficacy as an antivascular agent that reduced pericyte-driven in vitro angiogenesis, in contrast to paclitaxel that showed limited antiangiogenic activity [18]. Furthermore, eribulin treatment led to a specific gene signature that was different from that of paclitaxel in gene-expression profiling of drug-treated pericytes [18].

In preclinical studies, eribulin has a beneficial role in affecting EMT. In human breast cancer cells in vitro and xenografts in vivo, eribulin treatment was associated with a reversal of the EMT phenotype [20], which is associated with promotion of local invasiveness and metastasis [21]. Additionally, in an independent set of experiments, eribulin led to a decrease in observed cell migration and invasiveness in vitro and experimental metastasis in vivo [20]. As hypoxia is a key driver of the EMT and chemoresistance [21], the reduction in hypoxia observed with eribulin treatment may contribute to the observed reversal of EMT [3,22]. Interestingly, there is evidence for decreased Wnt3 


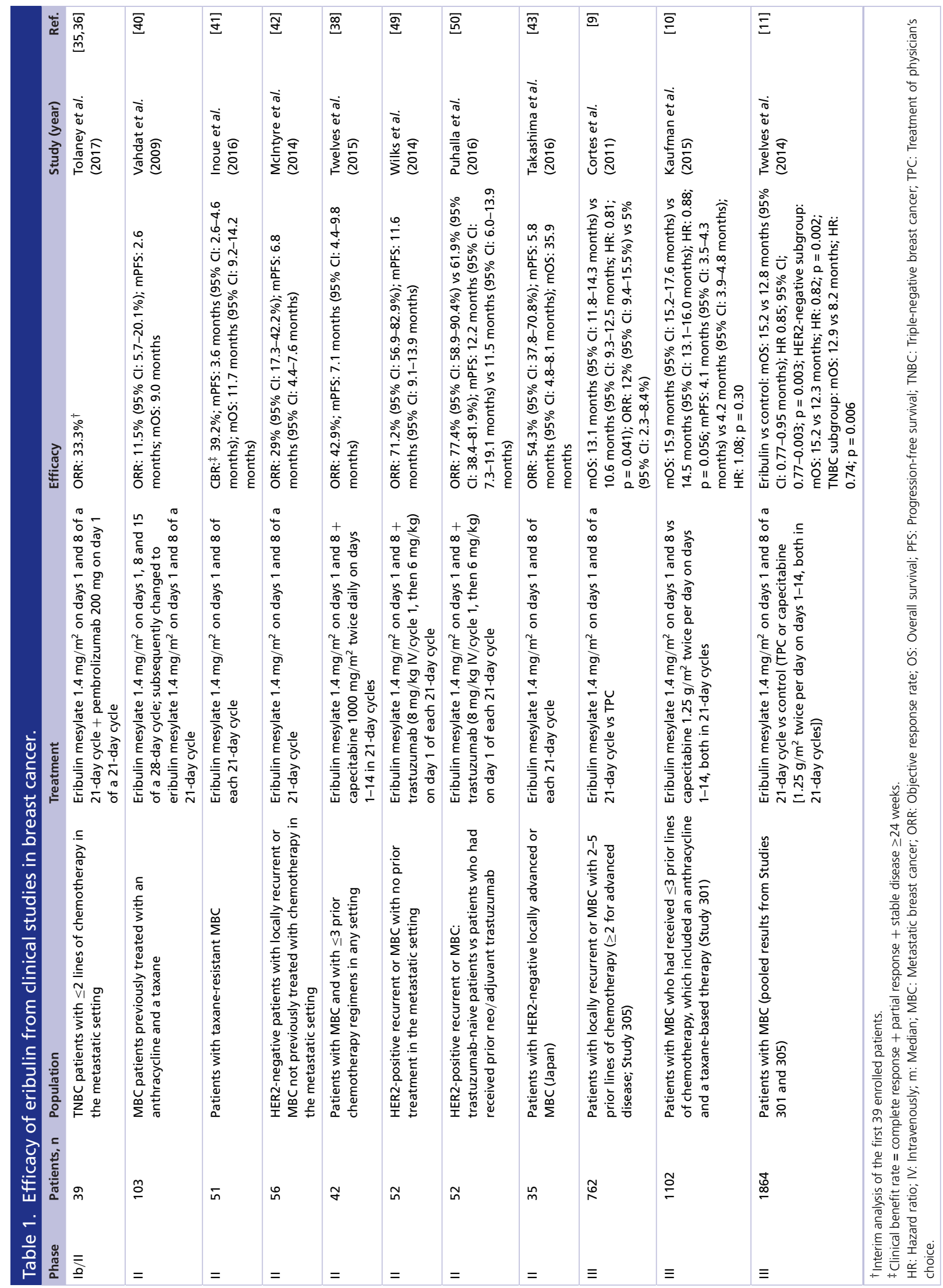


expression that supports reversal of EMT [23], and a recent preclinical study has shown that eribulin upregulates miR-195 expression and downregulates Wnt3a expression in nonbasal-like TNBC cells [24].

The effects of eribulin on EMT have been studied in models of breast cancer and other tumors [25]. A liquid biopsy method that enables quantitation of circulating tumor cells in EMT [26] may provide a way to investigate EMT in patients receiving eribulin. Suppression of EMT may be of therapeutic relevance for patients with breast cancer subtypes known for expressing high levels of genes involved in EMT such as the M subtype of TNBC [27].

One of the identified potential mechanisms of resistance to microtubule-targeting agents is constitutive activation of the PI3K/Akt survival pathway, either via mutational activation of the PI3K catalytic subunit (PIK3CA) or via inactivation of PTEN [28]. A recent analysis of PI3K mutations in more than 2000 breast cancer tumors showed that more than $50 \%$ had one or more alterations in the PI3K pathway (31\% PIK3CA mutated, 34\% PTEN lost and 5\% PTEN mutated) [29]. Eribulin has been shown to exhibit limited antitumor activity in PI3KCA-mutant xenograft models compared with PI3KCA-wild-type models [28]. However, coadministration of BKM120, a class 1 pan-PI3K inhibitor, with eribulin-induced marked tumor regression [28]. These observations support further studies of regimens that combine eribulin and PI3K inhibitors.

Preclinical studies of eribulin, in particular those focused on its nonmitotic MOA, may help shed light on the observed clinical outcomes with eribulin including the discordance between OS and progression-free survival (PFS) that was observed in the Phase III MBC trial of eribulin versus TPC [9]. In the primary analyses, no statistically significant difference in PFS was observed between eribulin and TPC despite a significant improvement in OS. Median PFS was 3.7 months with eribulin and 2.2 months with TPC (HR: 0.87; 95\% CI: 0.71-1.05; p = 0.137), whereas there was a significant increase in median OS for eribulin versus TPC (13.1 vs 10.6 months; HR: 0.81; 95\% CI: 0.66-0.99; $\mathrm{p}=0.041$ ) [9]. A similar trend was observed in the Phase III trial that evaluated eribulin versus capecitabine in patients with advanced or MBC [10]. In that study, median OS was numerically improved with eribulin compared with capecitabine (15.9 vs 14.5 months; HR: 0.88; 95\% CI: 0.77-1.00; $\mathrm{p}=0.056$ ); however, improvement in PFS was not observed between treatment arms (4.1 vs 4.2 months; HR: 1.08; 95\% CI: 0.93-1.25; $\mathrm{p}=0.30$ ). In a Phase III trial of patients with advanced soft tissue sarcoma, eribulin significantly improved OS compared with dacarbazine [30]. However, similar to the pivotal study in MBC, a significant OS benefit was observed despite a lack of statistically significant improvement in PFS. These observations raise the interesting possibility that eribulin-induced changes in tumor phenotype and vascularization seen in preclinical studies may contribute to a greater benefit of subsequent therapies and to improved OS outcomes; however, this hypothesis requires further investigation in controlled translational studies [31].

\section{Eribulin use in difficult-to-treat MBC}

TNBC is an aggressive breast cancer subtype that is associated with an increased risk of relapse, metastasis and poor clinical outcomes [32,33]. Subgroup analyses and pooled analyses from randomized Phase III trials of eribulin provide evidence for the efficacy of eribulin in patients with pretreated TNBC. In a prespecified subgroup analysis of Study 301, OS was found to be significantly longer for eribulin than capecitabine in certain patient subgroups [31]. In patients with TNBC, median OS was 14.4 versus 9.4 months (HR: 0.70; 95\% CI: 0.54-0.91; $\mathrm{p}=0.01$ ) [31]. Similarly, a pooled analysis of Phase III data from EMBRACE and Study 301 showed that eribulin significantly improved OS compared with control treatment in patients with TNBC (HR: 0.74; 95\% CI: 0.60-0.92; p $=0.006$ [11]. In a secondary pooled analysis, patients with TNBC from these studies who matched the European Union label of eribulin (i.e., those who had received at least one prior chemotherapy for advanced disease) also showed a significant OS benefit with eribulin versus the control arm (median OS: 12.4 vs 8.1 months; HR: 0.72; 95\% CI: 0.57-0.90; p < 0.01) [34].

In addition to pooled and subgroup analyses from randomized Phase III studies of eribulin $[11,31,34]$, there are emerging clinical data that demonstrate eribulin's therapeutic potential in TNBC. An open-label, single-arm, Phase $\mathrm{Ib} / \mathrm{II}$ study of eribulin in combination with the PD-1 antibody pembrolizumab compared the efficacy and safety in patients with metastatic TNBC [35]. In the Phase II portion of the study, patients were stratified by treatment line (i.e., first-line vs second- or third-line treatment in the metastatic setting) and outcomes were also evaluated by PDL1 tumor status [36]. In an interim analysis of 39 patients, the combination therapy showed an objective response rate (ORR) of $33 \%$ [36], which was higher than that observed historically with either eribulin or pembrolizumab as a monotherapy in patients with TNBC (ORR: 27.3 and 23\%, respectively) [22,37]. The combination also demonstrated comparable adverse events to those observed with either treatment as monotherapy [36]. 
In a Phase II study of patients with advanced breast cancer who had received prior anthracycline and taxane therapy, the combination of eribulin mesylate $\left(1.4 \mathrm{mg} / \mathrm{m}^{2}\right.$ on days 1 and 8 of a 21-day cycle) plus capecitabine showed promising antitumor activity (ORR: 42.9\%) [38], and greater activity than that of either drug administered as a single agent (ORR for eribulin, 11.5\%; ORR for capecitabine, 26\%) [39,40]. Eribulin and capecitabine are highly noncross-resistant to anthracycline/taxane treatment, which makes the combination regimen potentially promising for patients with aggressive estrogen receptor (ER)-positive or triple-negative disease. Although eribulin plus capecitabine has shown activity in the metastatic setting, there are currently no Phase III data available.

Eribulin has demonstrated clinical activity in patients whose disease had previously progressed on taxane-based therapies: a Phase II, single-arm, open-label, clinical trial in Japanese patients with well-defined taxane-resistant MBC showed that eribulin provided a clinical benefit rate (CBR) of 39\% and median OS of 11.7 months [41].

Eribulin treatment in the first-line setting in patients with HER2-negative MBC has also been investigated in Phase II trials. A Phase II trial of first-line eribulin treatment in patients with HER2-negative MBC reported a CBR of 52\%, median PFS of 6.8 months and an acceptable safety profile [42]. Similarly, a Phase II study in Japan in patients with HER2-negative locally advanced or MBC treated with eribulin as a first-line agent reported a CBR of $63 \%$ and median OS of 35.9 months [43]. A Phase II trial in Japan showed that eribulin exhibited efficacy and manageable toxicity in patients with pretreated HER2-negative MBC in first- to third-line use; first-line treatment led to a higher ORR and prolonged PFS and OS compared with second- or third-line treatment [44].

\section{Eribulin use in HER2 ${ }^{+}$breast cancer \& early-stage breast cancer}

There are data supporting eribulin's activity and tolerability in patients with HER2-positive MBC. The backbone of first-line treatment for HER2-positive breast cancer includes anti-HER2 agents (e.g., trastuzumab) [45]. The combination of trastuzumab with conventional chemotherapies has been evaluated in clinical trials and has shown efficacy for the treatment of HER2-positive MBC [46,47]. Eribulin has been evaluated in combination with trastuzumab in several clinical trials. A Phase I study demonstrated the safety of eribulin plus trastuzumab in patients with advanced or recurrent HER2-positive breast cancer [48]. In addition, a Phase II study of the combination of eribulin plus trastuzumab as first-line treatment in HER2-positive MBC reported an ORR of 71.2\% and median PFS of 11.6 months [49]. Similarly, another Phase II trial showed that first- line eribulin/trastuzumab treatment in patients with HER2-positive MBC resulted in antitumor activity and was well-tolerated, regardless of prior (neo)adjuvant trastuzumab treatment [50]. A Phase II trial of eribulin in combination with trastuzumab and pertuzumab showed that the combination was well-tolerated and may represent a treatment strategy for taxane-pretreated, HER2-positive, advanced breast cancer [51].

Evidence for the use of eribulin in earlier-stage breast cancer is more limited than in metastatic disease and later lines of treatment. A Phase II neoadjuvant trial of eribulin in combination with carboplatin in patients with TNBC was well-tolerated and showed promising clinical activity. A majority of patients $(24 / 29 ; 83 \%)$ achieved a clinical complete or partial response and 43\% (13/30) of patients achieved a pathologic complete response [52]. A feasibility trial evaluated the combination of eribulin mesylate $1.4 \mathrm{mg} / \mathrm{m}^{2}$ on days 1 and 8 of a 21-day cycle plus capecitabine $\left(900 \mathrm{mg} / \mathrm{m}^{2}\right.$ twice daily on days $1-14$ of a 21 -day cycle) as adjuvant therapy in postmenopausal women $(\mathrm{n}=67)$ with ER-positive early-stage breast cancer [53]. In this study, the combination regimen was feasible and the safety profiles were similar to known toxicities of both monotherapies.

\section{Observational studies of eribulin}

Observational 'real-world' studies have reported efficacy and tolerability results for eribulin treatment consistent with those reported in clinical trials (Table 2). Clinical benefit has been reported in a large $(\mathrm{N}=574)$ unselected population with MBC [54], heavily pretreated patients with MBC [55], elderly patients $\geq 70$ years old [56], heavily pretreated elderly patients with locally recurrent/MBC [57], metastatic TNBC [58,59], ER-positive MBC [60], hormone-refractory breast cancer [61], taxane-refractory MBC [62] and in patients with brain metastases [63,64]. In a retrospective, real-world study of 252 patients with metastatic TNBC (Table 2), it was noted that the estimated real-world OS of 14.7 months for late-line eribulin users was consistent with the 13.1 months reported in EMBRACE [59,65].

In a retrospective analysis of data from Study 301 (Table 2), six patients with brain metastases (three each treated with eribulin or capecitabine) were analyzed as a case series [64]. All three patients treated with eribulin received six cycles and had some degree of reduction in the size of their brain lesions during treatment. The three patients on capecitabine had varied outcomes regarding brain metastases: one patient had an increase in the size of brain 


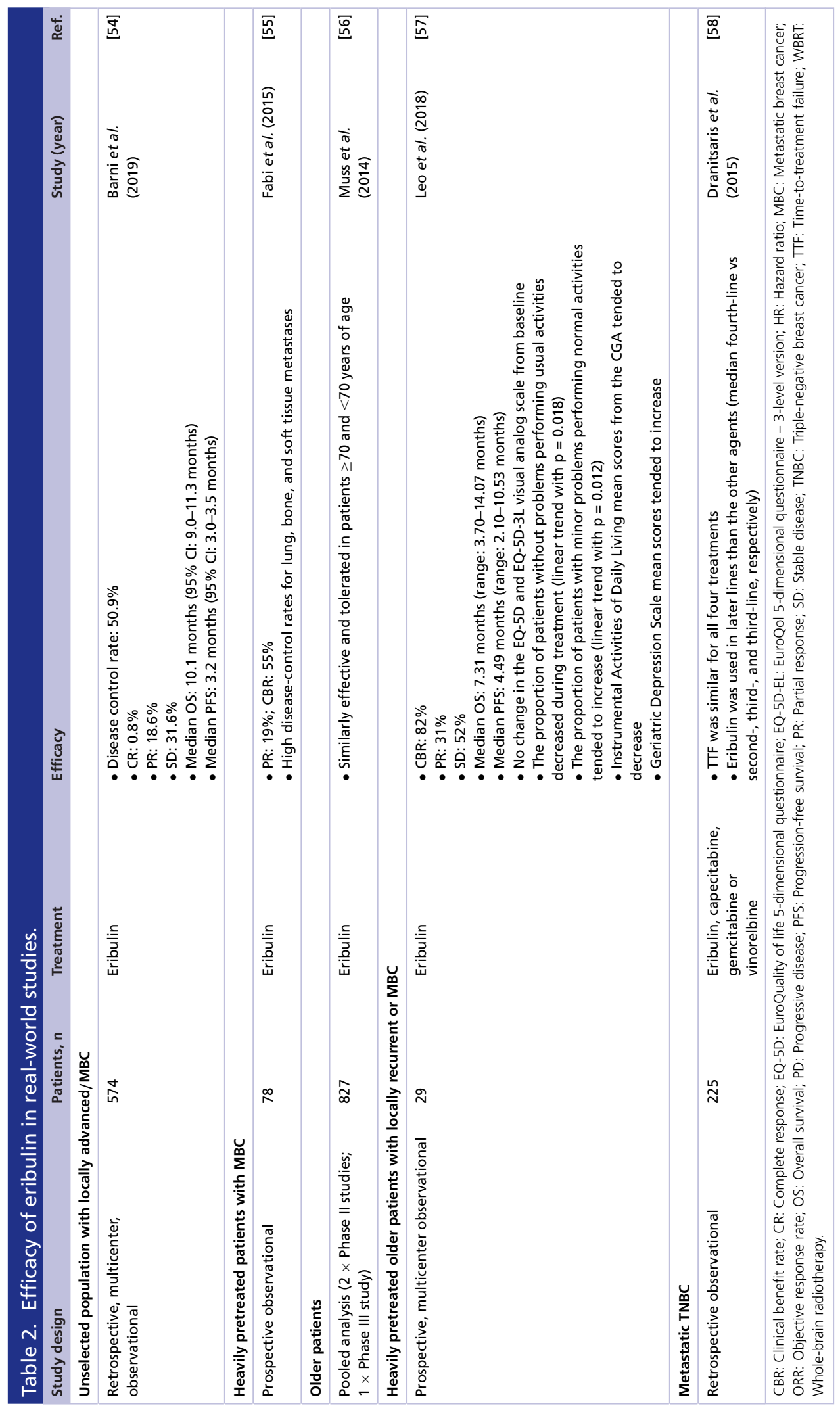




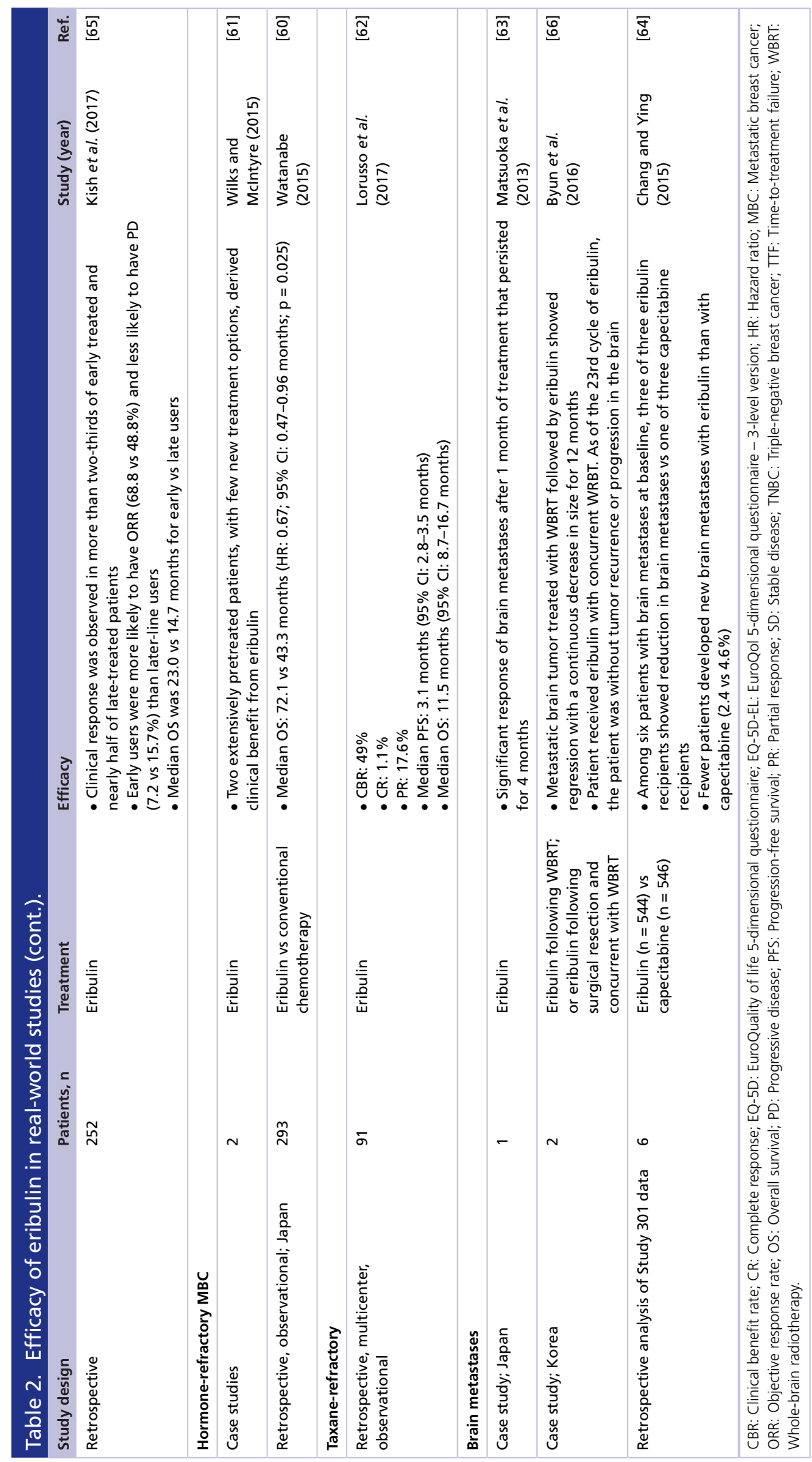


lesions; one patient had no observable change in the size of brain lesions; and one patient had a complete response that was not confirmed with a follow-up scan.

A recent case study report of two patients with breast cancer and brain metastases showed that eribulin treatment, either following whole-brain radiotherapy (WBRT) or surgical resection of the tumor, resulted in clinical regression [66]. One patient (with HER2-negative breast cancer) received WBRT followed by eribulin monotherapy. After eight cycles of treatment, the metastatic brain tumors showed regression with systematically stable disease. The second patient (hormone receptor-negative, HER2-positive breast cancer) received eribulin with concurrent WBRT after surgical resection of the tumor. After 23 cycles of eribulin, the patient was without tumor recurrence or progression in the brain [66]. Although suggestive of a clinical benefit to patients with breast cancer who develop brain metastases, future studies are needed to evaluate the effects of eribulin in this setting.

\section{Safety profile}

In clinical studies, two of the most common adverse events associated with eribulin included neutropenia and neuropathy [4]. In the pivotal EMBRACE study, which included patients with heavily pretreated MBC, neutropenia occurred in $52 \%$ of patients in the eribulin arm and was managed with dose delays, reductions and the administration of growth factors (granulocyte colony-stimulating factor in $18 \%$ of patients) [9]. The incidence of severe neutropenia (absolute neutophil count $<500 / \mathrm{mm}^{3}$ ) lasting more than 1 week occurred in 12\% (62/503) of patients and led to treatment discontinuation in less than $1 \%$ of patients [4]. Febrile neutropenia occurred in 5\% (23/503) of patients, with two patients (0.4\%) dying from complications of febrile neutropenia [4]. Grade $3 / 4$ neutropenia has also been reported in real-world studies of eribulin in patients with heavily pretreated $\mathrm{MBC}$ with rates ranging from $22 \%$ $(17 / 78)$ to $49 \%(404 / 827)$ [55,56]. An unselected population of patients receiving eribulin in a real-world setting were reported with grade $3 / 4$ neutropenia in $12.2 \%$ (70/574) [54]. In EMBRACE, peripheral neuropathy was the most common toxicity leading to treatment discontinuation (5\% of patients; $24 / 503$ ), with grade 3 peripheral neuropathy occurring in $8 \%(40 / 503)$ of patients and grade 4 in $0.4 \%(2 / 503)$ of patients [4]. Grade $3 / 4$ peripheral neuropathy has also been reported at rates of $5 \%(4 / 78)$ to $7 \%(57 / 827)$ of patients among those with heavily pretreated MBC [55,56]. In an unselected population, the rate of grade 3/4 neuropathy was $2.6 \%(15 / 574)$ [54].

Alternative schedules of eribulin administration are being evaluated with the goal of enabling patients who are sensitive to myelosuppression to better tolerate treatment. Compared with the licensed dosing schedule of eribulin administered on days 1 and 8 every 3 weeks, a modified biweekly regimen provides additional time for hematologic recovery between treatment administrations, which may potentially improve eribulin's safety profile [67]. In a prospective Phase II trial in Japan in patients with pretreated MBC, a biweekly schedule of eribulin $\left(1.4 \mathrm{mg} / \mathrm{m}^{2}\right.$ on days 1 and 15 of a 28-day cycle) was tolerable and had comparable efficacy in patients who were intolerant of the standard eribulin schedule. In that study, patients on the biweekly schedule had a mean relative dose intensity of $62.7 \%$, ORR of $21 \%$ and a median OS of 523 days [68]. A Phase II trial in the USA evaluating an eribulin biweekly schedule in patients with HER2-negative MBC has recently been completed and should provide additional insight into the efficacy and safety of eribulin treatment using an alternative dosing schedule.

A Phase II trial in patients with MBC evaluating the incidence of peripheral neuropathy with standard-regimen eribulin versus ixabepilone demonstrated that, although not statistically significantly different, fewer patients receiving eribulin discontinued treatment due to neuropathy than those who received ixabepilone (3.9 vs 18.0\%) [69]. Time to onset of neuropathy was 35.9 weeks for eribulin compared with 11.6 weeks for ixabepilone. Taken together, these results demonstrate that neuropathy associated with eribulin tended to occur later and patients treated with eribulin remained on treatment longer.

The presence of liver metastasis is often a challenge for patients with breast cancer receiving treatment in later-line settings. Pharmacokinetic studies have demonstrated that eribulin is predominantly excreted unchanged (i.e., not metabolized), with only 5-6\% of the administered dose eliminated through the urine [70,71]. Based on findings from a Phase I pharmacokinetic study in patients with solid tumors and mild-to-moderate liver impairment [72], eribulin dose reductions are recommended [4]. Liver impairment increased exposure to eribulin in patients with Child-Pugh class A impairment (1.75-fold higher) and Child-Pugh class B impairment (2.48-fold higher) [72]. Further analyses are needed to help determine the safety and efficacy of eribulin in patients with $\mathrm{MBC}$ and liver impairment. 


\section{Conclusion}

Eribulin remains a unique anticancer agent, with both mitotic and nonmitotic MOAs, for the treatment of MBC after failure of an anthracycline and a taxane. Clinical trial data from Phase III studies have shown that eribulin has activity in difficult-to-treat patients, including those with pretreated HER2-negative disease or TNBC. Safety data are consistent with other microtubule-targeting antimitotic agents, and additional safety analyses highlight the potential of eribulin treatment in specific patient populations. Combination studies of eribulin with immunotherapies and established cytotoxic agents are ongoing and may help to shape the landscape of future treatments for MBC.

\section{Future perspective}

While the cytotoxic agent landscape remains relatively static, the use of combination regimens and immunotherapies for treating patients with advanced TNBC appears very promising. Results from the Phase II neoadjuvant trial of eribulin and carboplatin in early-stage TNBC were encouraging, showing pathologic complete response in $43.3 \%$ of patients, and $80.0 \%$ of patients achieving complete response or partial response [52]. In addition, the combination of eribulin plus pembrolizumab demonstrated activity in a Phase Ib/II trial in patients with metastatic TNBC [35], warranting further clinical evaluation of the combination. The results of ongoing clinical trials of immunotherapies in combination with cytotoxic therapies will help dictate the changing landscape of combination therapies for TNBC and other difficult-to-treat breast cancers. As an example, a recent Phase I trial of eribulin in combination with the C-X-C chemokine receptor type 4 antagonist, balixafortide, showed promising preliminary activity in patients with heavily pretreated HER2-negative MBC [73] and the combination has received fast-track designation by the US FDA. Therefore, there is continued interest in the clinical development of eribulin-based combination regimens for patients with $\mathrm{MBC}$.

\section{Executive summary}

\section{Advances in understanding eribulin's mechanism of action}

- Eribulin is a potent microtubule dynamics inhibitor that prevents mitotic spindle formation, resulting in mitotic arrest and apoptosis.

- Eribulin also exhibits nonmitotic activity including vascular remodeling, reversal of the epithelial-to-mesenchymal phenotype and suppression of cancer cell migration, invasion and experimental metastasis.

Eribulin use in difficult-to-treat metastatic breast cancer

- Eribulin demonstrated statistically significant improvements in overall survival in patients with triple-negative breast cancer (TNBC) in subgroup and pooled analyses from two randomized Phase III trials.

- Combination studies showed promising clinical activity in patients with estrogen receptor (ER)-positive or TNBC.

- Several Phase II studies demonstrated encouraging antitumor activity in patients with taxane-resistant metastatic breast cancer (MBC) or HER2-negative MBC.

Eribulin use in HER2-positive breast cancer \& early-stage breast cancer

- The eribulin and trastuzumab combination regimen was effective and well tolerated in patients with HER2-positive breast cancer.

- Additional eribulin combination therapies demonstrated clinical activity and an acceptable safety profile in patients with early-stage TNBC and ER-positive breast cancer.

\section{Observational studies of eribulin}

- Eribulin provided a clinical benefit in a number of observational studies for heavily pretreated patients with MBC, elderly patients $\geq 70$ years old, metastatic TNBC, ER-positive MBC, hormone-refractory breast cancer and in patients with brain metastases.

\section{Safety profile}

- Neutropenia and neuropathy are the most common adverse events associated with eribulin.

- Neutropenia was managed with supportive medications and dose modifications. A Phase II trial showed that fewer patients receiving eribulin discontinued treatment due to neuropathy than patients receiving ixabepilone (3.9 vs $18.0 \%$ ).

- Eribulin dose reductions are recommended for patients with mild-to-moderate hepatic impairment. 
Financial \& competing interests disclosure

This work was supported by Eisai Inc which provided funding to Oxford PharmaGenesis Inc., for medical writing and editing assistance with this manuscript. The authors had full control of the content and received no other financial assistance. Eisai did not take part in designing the concept, drafting the content, nor in the decision to submit for publication. J O'Shaughnessy received funding from consulting: AstraZeneca, Celgene, Eli-Lilly, LaRoche, Lilly USA, Merck, Novartis and Pfizer; honoraria: Seattle Genetics; speaking fees: Blue Earth Diagnostics, Novartis; vendor-sponsored travel: AstraZeneca, Blue Earth Diagnostics, Eli-Lilly, Merck, Novartis, Seattle Genetics. V Kaklamani received funding from speakers' bureau: Eisai, Genomic Health, Genentech, Pfizer, Novartis; grants: Eisai; consulting: Athenex, Pfizer, Novartis. K Kalinsky received funding from consulting: Biotheranostics, Eli-Lilly, Pfizer, Amgen, Novartis, Eisai, AstraZeneca, Odonate Therapeutics, Ipsen, Genentech; speakers' bureau: Eli-Lilly; institutional support: Incyte, Genentech, Eli-Lilly, Pfizer, Calithera Biosciences, Acetylon, Seattle Genetics, Amgen, Zeno Pharmaceuticals, CytomX Therapeutics; spouse: employment at Array Biopharma; vendor-sponsored travel: Eli-Lilly, Novartis, Genentech, Ipsen and Amgen. The authors have no other relevant affiliations or financial involvement with any organization or entity with a financial interest in or financial conflict with the subject matter or materials discussed in the manuscript apart from those disclosed.

Medical writing support was provided by Eli Berdougo of Oxford PharmaGenesis Inc., PA, USA, and was funded by Eisai Inc., NJ, USA.

\section{Open access}

This work is licensed under the Attribution-NonCommercial-NoDerivatives 4.0 Unported License. To view a copy of this license, visit http://creativecommons.org/licenses/by-nc-nd/4.0/

\section{References}

Papers of special note have been highlighted as: $\bullet$ of interest; $\bullet \bullet$ of considerable interest

1. National Cancer Institute. SEER Cancer Statistics Factsheets: female breast cancer. http://seer.cancer.gov/statfacts/html/breast.html

2. Towle MJ, Salvato KA, Budrow J et al. In vitro and in vivo anticancer activities of synthetic macrocyclic ketone analogues of halichondrin B. Cancer Res. 61(3), 1013-1021 (2001).

- Demonstrates the highly potent in vitro and in vivo anticancer activities of eribulin, a fully synthetic, macrocyclic ketone analog of halichondrin $\mathrm{B}$.

3. Dybdal-Hargreaves NF, Risinger AL, Mooberry SL. Eribulin mesylate: mechanism of action of a unique microtubule-targeting agent. Clin. Cancer Res. 21(11), 2445-2452 (2015).

4. Halaven (eribulin mesylate) injection, for intravenous use [prescribing information]. Eisai Inc., NJ, USA (2016).

5. Halaven $0.44 \mathrm{mg} / \mathrm{ml}$ solution for injection [summary of product characteristics]. Eisai Europe Limited, Hertfordshire, UK (2017) www.medicines.org.uk/emc/product/4517/smpc

6. Jordan MA, Kamath K, Manna T et al. The primary antimitotic mechanism of action of the synthetic halichondrin E7389 is suppression of microtubule growth. Mol. Cancer Ther. 4(7), 1086-1095 (2005).

- Demonstrates unique aspects of microtubule dynamics inhibition of eribulin via binding to exposed $\beta$-tubulin subunits at microtubule plus ends.

7. Okouneva T, Azarenko O, Wilson L, Littlefield BA, Jordan MA. Inhibition of centromere dynamics by eribulin (E7389) during mitotic metaphase. Mol. Cancer Ther. 7(7), 2003-2011 (2008).

8. Smith JA, Wilson L, Azarenko $\mathrm{O}$ et al. Eribulin binds at microtubule ends to a single site on tubulin to suppress dynamic instability. Biochemistry 49(6), 1331-1337 (2010).

9. Cortes J, O'Shaughnessy J, Loesch D et al. Eribulin monotherapy versus treatment of physician's choice in patients with metastatic breast cancer (EMBRACE): a Phase III open-label randomized study. Lancet 377(9769), 914-923 (2011).

10. Kaufman PA, Awada A, Twelves C et al. Phase III open-label randomized study of eribulin mesylate versus capecitabine in patients with locally advanced or metastatic breast cancer previously treated with an anthracycline and a taxane. J. Clin. Oncol. 33(6), 594-601 (2015).

11. Twelves C, Cortes J, Vahdat L et al. Efficacy of eribulin in women with metastatic breast cancer: a pooled analysis of two Phase III studies. Breast Cancer Res. Treat. 148(3), 553-561 (2014).

-. This pooled analysis of Study 305 and Study 301 confirmed the significant survival benefit of eribulin compared with control following treatment with a taxane and an anthracycline in either the adjuvant or the metastatic setting.

12. Dranitsaris G, Beegle N, Kalberer T, Blau S, Cox D, Faria C. A comparison of toxicity and health care resource use between eribulin, capecitabine, gemcitabine, and vinorelbine in patients with metastatic breast cancer treated in a community oncology setting. J. Oncol. Pharm. Pract. 21(3), 170-177 (2015).

13. Doodhi H, Prota AE, Rodríguez-García R et al. Termination of protofilament elongation by eribulin induces lattice defects that promote microtubule catastrophes. Curr. Biol. 26(13), 1713-1721 (2016).

14. Cheng KL, Bradley T, Budman DR. Novel microtubule-targeting agents - the epothilones. Biologics 2(4), 789-811 (2008). 
15. Jain S, Vahdat LT. Eribulin mesylate. Clin. Cancer Res 17(21), 6615-6622 (2011).

16. Kuznetsov G, Towle MJ, Cheng $\mathrm{H}$ et al. Induction of morphological and biochemical apoptosis following prolonged mitotic blockage by halichondrin B macrocyclic ketone analog E7389. Cancer Res. 64(16), 5760-5766 (2004).

17. Chanez B, Gonçalves A, Badache A, Verdier-Pinard P. Eribulin targets a ch-TOG-dependent directed migration of cancer cells. Oncotarget 6(39), 41667-41678 (2015).

18. Agoulnik SI, Kawano S, Taylor N et al. Eribulin mesylate exerts specific gene expression changes in pericytes and shortens pericyte-driven capillary network in vitro. Vasc. Cell 6(1), 3 (2014).

19. Funahashi $Y$, Okamoto $\mathrm{K}$, Adachi $\mathrm{Y}$ et al. Eribulin mesylate reduces tumor microenvironment abnormality by vascular remodeling in preclinical human breast cancer models. Cancer Sci. 105(10), 1334-1342 (2014).

20. Yoshida T, Ozawa Y, Kimura T et al. Eribulin mesilate suppresses experimental metastasis of breast cancer cells by reversing phenotype from epithelial-mesenchymal transition (EMT) to mesenchymal-epithelial transition (MET) states. Br. J. Cancer 110(6), 1497-1505 (2014).

21. Jung HY, Fattet L, Yang J. Molecular pathways: linking tumor microenvironment to epithelial-mesenchymal transition in metastasis. Clin. Cancer Res. 21(5), 962-968 (2015).

22. Kashiwagi S, Fukushima W, Asano Y et al. Identification of predictive markers of the therapeutic effect of eribulin chemotherapy for locally advanced or metastatic breast cancer. BMC Cancer 17(1), 604 (2017).

23. Wu Y, Ginther C, Kim J et al. Expression of Wnt3 activates Wnt/ $\beta$-catenin pathway and promotes EMT-like phenotype in trastuzumab-resistant HER2-overexpressing breast cancer cells. Mol. Cancer Res. 10(12), 1597-1606 (2012).

24. Furuya K, Sasaki A, Tsunoda Y et al. Eribulin upregulates miR-195 expression and downregulates Wnt3a expression in non-basal-like type of triple-negative breast cancer cell MDA-MB-231. Hum. Cell 29(2), 76-82 (2016).

25. Cortes J, Schöffski P, Littlefield BA. Multiple modes of action of eribulin mesylate: emerging data and clinical implications. Cancer Treat. Rev. 70, 190-198 (2018).

- Discusses the mitotic and nonmitotic effects of eribulin on tumor biology, evidence from the clinical setting supporting eribulin's nonmitotic effects and potential biomarkers for eribulin response.

26. Bulfoni M, Gerratana L, Del Ben F et al. In patients with metastatic breast cancer the identification of circulating tumor cells in epithelial-to-mesenchymal transition is associated with a poor prognosis. Breast Cancer Res. 18(1), 30 (2016).

27. Gerratana L, Basile D, Buono G et al. Androgen receptor in triple negative breast cancer: a potential target for the targetless subtype. Cancer Treat. Rev. 68, 102-110 (2018).

28. Serra V, Gris-Oliver A, Saura C et al. PI3K blockade enhances the antitumor activity of eribulin in PIK3CA-mutant eribulin-resistant tumor xenografts [abstract]. Cancer Res. 73(24 Suppl.), Abstract P5-08-06 (2013).

29. Millis SZ, Ikeda S, Reddy S, Gatalica Z, Kurzrock R. Landscape of phosphatidylinositol-3-kinase pathway alterations across 19784 diverse solid tumors. JAMA Oncol. 2(12), 1565-1573 (2016).

30. Schöffski P, Chawla S, Maki RG et al. Eribulin versus dacarbazine in previously treated patients with advanced liposarcoma or leiomyosarcoma: a randomized, open-label, multicentre, Phase III trial. Lancet 387(10028), 1629-1637 (2016).

31. Twelves C, Awada A, Cortes J et al. Subgroup analyses from a Phase III, open-label, randomized study of eribulin mesylate versus capecitabine in pretreated patients with advanced or metastatic breast cancer. Breast Cancer (Auckl.) 10, 77-84 (2016).

32. Penault-Llorca F, Viale G. Pathological and molecular diagnosis of triple-negative breast cancer: a clinical perspective. Ann. Oncol. 23(Suppl. 6), vi19-vi22 (2012).

33. Torzilli PA, Bourne JW, Cigler T, Vincent CT. A new paradigm for mechanobiological mechanisms in tumor metastasis. Semin. Cancer Biol. 22(5-6), 385-395 (2012).

34. Pivot X, Marmé F, Koenigsberg R, Guo M, Berrak E, Wolfer A. Pooled analyses of eribulin in metastatic breast cancer patients with at least one prior chemotherapy. Ann. Oncol. 27(8), 1525-1531 (2016).

- Reports on a pooled analysis from Study 305 and Study 301, which demonstrates that eribulin increased survival rates compared with control treatment in patients with metastatic breast cancer $(\mathrm{MBC})$ who matched the EU label.

35. Tolaney S, Savulsky C, Aktan G et al. Phase Ib/II study to evaluate eribulin mesylate in combination with pembrolizumab in patients with metastatic triple-negative breast cancer [abstract]. Cancer Res. 77(4 Suppl.), Abstract P5-15-02 (2017).

- This report describes the interim analysis of a Phase Ib/II study of the novel regimen of eribulin + pembrolizumab in patients with triple-negative breast cancer. The study provides the first evidence of objective responses, including complete responses, along with safety and tolerability data with this combination in these patients.

36. Tolaney SM, Savulsky C, Aktan G et al. Phase Ib/II study to evaluate eribulin mesylate in combination with pembrolizumab in patients with metastatic triple-negative breast cancer. Presented at: European Cancer Congress. Amsterdam, The Netherlands, 27-30 January 2017.

37. Adams S, Loi S, Toppmeyer D et al. Phase II study of pembrolizumab as first-line therapy for PD-L1-positive metastatic triple-negative breast cancer (mTNBC): preliminary data from KEYNOTE-086 cohort B [abstract]. J. Clin. Oncol. 35 (15 Suppl.), Abstract 1088 (2017). 
38. Twelves C, Nasim MY, Anthoney A, Savulsky CI, Yin S, Evans TRJ. Efficacy and safety of eribulin in combination with capecitabine in patients with metastatic breast cancer: an open-label, Phase II dose-confirmation study [abstract]. Cancer Res. 75 (9 Suppl.), Abstract P3-13-04 (2015).

39. Blum JL, Dieras V, Lo Russo PM et al. Multicenter, Phase II study of capecitabine in taxane-pretreated metastatic breast carcinoma patients. Cancer 92(7), 1759-1768 (2001).

40. Vahdat LT, Pruitt B, Fabian CJ et al. Phase II study of eribulin mesylate, a halichondrin B analog, in patients with metastatic breast cancer previously treated with an anthracycline and a taxane. J. Clin. Oncol. 27(18), 2954-2961 (2009).

41. Inoue $\mathrm{K}$, Saito $\mathrm{T}$, Okubo $\mathrm{K}$ et al. Phase II clinical study of eribulin monotherapy in Japanese patients with metastatic breast cancer who had well-defined taxane resistance. Breast Cancer Res. Treat. 157(2), 295-305 (2016).

42. McIntyre K, O’Shaughnessy J, Schwartzberg L et al. Phase II study of eribulin mesylate as first-line therapy for locally recurrent or metastatic human epidermal growth factor receptor 2-negative breast cancer. Breast Cancer Res. Treat. 146(2), 321-328 (2014).

43. Takashima T, Tokunaga S, Tei $S$ et al. A Phase II, multicenter, single-arm trial of eribulin as first-line chemotherapy for HER2-negative locally advanced or metastatic breast cancer. Springerplus 5, 164 (2016).

44. Maeda S, Saimura M, Minami S et al. Efficacy and safety of eribulin as first- to third-line treatment in patients with advanced or metastatic breast cancer previously treated with anthracyclines and taxanes. Breast 32, 66-72 (2017).

45. National Comprehensive Cancer Network Clinical Practice Guidelines in Oncology (NCCN Guidelines ${ }^{\circledR}$ ). Breast cancer. Version 1.2018. www.nccn.org/professionals/physician_gls/pdf/breast.pdf

46. Ishida T, Kiba T, Takeda M et al. Phase II study of capecitabine and trastuzumab combination chemotherapy in patients with HER2 overexpressing metastatic breast cancers resistant to both anthracyclines and taxanes. Cancer Chemother. Pharmacol. 64(2), 361-369 (2009).

47. Heinemann V, Di Gioia D, Vehling-Kaiser U et al. A prospective multicenter Phase II study of oral and i.v. vinorelbine plus trastuzumab as first-line therapy in HER2-overexpressing metastatic breast cancer. Ann. Oncol. 22(3), 603-608 (2011).

48. Mukai H, Saeki T, Shimada K et al. Phase I combination study of eribulin mesylate with trastuzumab for advanced or recurrent human epidermal growth factor receptor 2 positive breast cancer. Invest. New Drugs 33(1), 119-127 (2015).

49. Wilks S, Puhalla S, O'Shaughnessy J et al. Phase II, multicenter, single-arm study of eribulin mesylate with trastuzumab as first-line therapy for locally recurrent or metastatic HER2-positive breast cancer. Clin. Breast Cancer 14(6), 405-412 (2014).

50. Puhalla S, Wilks S, Brufsky AM et al. Clinical effects of prior trastuzumab on combination eribulin mesylate plus trastuzumab as first-line treatment for human epidermal growth factor receptor 2 positive locally recurrent or metastatic breast cancer: results from a Phase II, single-arm, multicenter study. Breast Cancer (Dove Med. Press) 8, 231-239 (2016).

51. Araki K, Fukada I, Kobayashi K, Takahashi S, Ito Y. Eribulin should be a candidate strategy in combination with pertuzumab plus trastuzumab for taxane pretreated HER2 positive advance breast cancer [abstract]. Cancer Res. 77(4 Suppl.), Abstract P5-15-11 (2017).

52. Kaklamani VG, Jeruss JS, Hughes E et al. Phase II neoadjuvant clinical trial of carboplatin and eribulin in women with triple negative early-stage breast cancer (NCT01372579). Breast Cancer Res. Treat. 151(3), 629-638 (2015).

53. Smith JW 2nd, Vukelja S, Hoffman AD et al. Phase II, multicenter, single-arm, feasibility study of eribulin combined with capecitabine for adjuvant treatment in estrogen receptor-positive, early-stage breast cancer. Clin. Breast Cancer 16(1), 31-37 (2016).

54. Barni S, Livraghi L, Morritti M et al. Eribulin in the treatment of advanced breast cancer: real-world scenario from 39 Italian centers ESEMPiO study. Future Oncol. 15(1), 33-44 (2019).

55. Fabi A, Moscetti L, Ciccarese $M$ et al. Eribulin in heavily pretreated metastatic breast cancer patients and clinical/biological feature correlations: impact on the practice. Future Oncol. 11(3), 431-438 (2015).

56. Muss H, Cortes J, Vahdat LT et al. Eribulin monotherapy in patients aged 70 years and older with metastatic breast cancer. Oncologist 19(4), 318-327 (2014).

- Describes a pooled analysis of data from two single-arm Phase II studies and one open-label randomized Phase III study for selected elderly patients with MBC and good baseline performance status. The analysis showed that eribulin monotherapy resulted in overall survival, progression-free survival, clinical benefit rate and tolerability for the elderly patients similar to those of younger patients in the studies.

57. Leo S, Arnoldi E, Repetto L et al. Eribulin mesylate as third or subsequent line chemotherapy for elderly patients with locally recurrent or metastatic breast cancer: a multicentric observational study of GIOGer (Italian Group of Geriatric Oncology)-ERIBE. Oncologist doi:10.1634/theoncologist.2017-0676 (2018) (Epub ahead of print).

58. Dranitsaris G, Gluck S, Faria C, Cox D, Rugo H. Comparative effectiveness analysis of monotherapy with cytotoxic agents in triple-negative metastatic breast cancer in a community setting. Clin. Ther. 37(1), 134-144 (2015).

59. Kish JK, Mougalian SS, Copher R, McAllister L, Zhixiao W, Broscious M. Utilization and outcomes of eribulin in triple negative metastatic breast cancer: real-world findings [abstract]. Cancer Res. 77(4 Suppl.), Abstract P5-15-16 (2017).

60. Watanabe J. Eribulin monotherapy improved survivals in patients with ER-positive HER2-negative metastatic breast cancer in the real world: a single institutional review. Springerplus 4, 625 (2015). 
61. Wilks S, McIntyre K. Case studies in the management of metastatic breast cancer with eribulin. Clin. Med. Insights Oncol. 9, 81-85 (2015).

62. Lorusso V, Cinieri S, Latorre A et al. Efficacy and safety of eribulin in taxane-refractory patients in the 'real world'. Future Oncol. 13(11), 971-978 (2017).

63. Matsuoka H, Tsurutani J, Tanizaki J et al. Regression of brain metastases from breast cancer with eribulin: a case report. BMC Res. Notes 6, 541 (2013).

64. Chang AY, Ying XX. Brain metastases from breast cancer and response to treatment with eribulin: a case series. Breast Cancer (Auckl.) 9 , 19-24 (2015).

65. Kish JK, Mougalian SS, Copher R, McAllister L, Wang Z, Broscious M. Utilization and outcomes of eribulin in triple negative metastatic breast cancer: real-world findings. Poster presented at: San Antonio Breast Cancer Symposium (SABCS). TX, USA, 6-10 December (2016).

66. Byun KD, Ahn SG, Baik HJ et al. Eribulin mesylate combined with local treatment for brain metastasis from breast cancer: two case reports. J. Breast Cancer 19(2), 214-217 (2016).

67. Yoshinami T, Masuda N, Nakayama T et al. The utility of bi-weekly eribulin therapy for metastatic breast cancer: a Japanese multicenter Phase II study (JUST-STUDY) [abstract]. J. Clin. Oncol. 33(15 Suppl.), Abstract 1026 (2015).

68. Ohtani S, Nakayama T, Yoshinami T et al. Bi-weekly eribulin therapy for metastatic breast cancer: a multicenter Phase II prospective study (JUST-STUDY). Breast Cancer 25(4), 438-446 (2018).

69. Vahdat LT, Garcia AA, Vogel C et al. Eribulin mesylate versus ixabepilone in patients with metastatic breast cancer: a randomized Phase II study comparing the incidence of peripheral neuropathy. Breast Cancer Res. Treat. 140(2), 341-351 (2013).

70. Goel S, Mita AC, Mita M et al. A Phase I study of eribulin mesylate (E7389), a mechanistically novel inhibitor of microtubule dynamics, in patients with advanced solid malignancies. Clin. Cancer Res. 15(12), 4207-4212 (2009).

71. Tan AR, Rubin EH, Walton DC et al. Phase I study of eribulin mesylate administered once every 21 days in patients with advanced solid tumors. Clin. Cancer Res. 15(12), 4213-4219 (2009).

72. Devriese LA, Witteveen PO, Marchetti $S$ et al. Pharmacokinetics of eribulin mesylate in patients with solid tumors and hepatic impairment. Cancer Chemother. Pharmacol. 70(6), 823-832 (2012).

73. Pernas S, Martin M, Kaufman PA et al. Balixafortide plus eribulin in HER2-negative metastatic breast cancer: a Phase I, single-arm, dose-escalation trial. Lancet Oncol. 19(6), 812-824 (2018).

- Reports the results of a Phase I trial of eribulin in combination with the CXCR4 antagonist, balixafortide. Based on the results of this study, the US FDA granted a fast-track designation to the combination for the treatment of patients with HER2-negative $\mathrm{MBC}$ who regressed after treatment with $\geq 2$ chemotherapies in the metastatic setting. 
